Detailed performance study of ATLAS endcap muon trigger with beam collision

This article has been downloaded from IOPscience. Please scroll down to see the full text article. 2010 JINST 5 C11010

(http://iopscience.iop.org/1748-0221/5/11/C11010)

View the table of contents for this issue, or go to the journal homepage for more

Download details:

IP Address: 137.138.125.164

The article was downloaded on 10/01/2011 at $13: 13$

Please note that terms and conditions apply. 


\title{
Detailed performance study of ATLAS endcap muon trigger with beam collision
}

\author{
T. Hayakawa ${ }^{1}$ \\ Graduate school of Science, Kobe University, \\ 1-1 Rokkodai, Nada-Kobe, Japan \\ E-mail: takashi.hayakawa@cern.ch
}

ABSTRACT: Since the first p-p collision of the LHC, The Trigger System has been providing level1 trigger signals of muon candidates with the specified latency to the central trigger system. The timing calibration is a vital issue to identify the bunch crossing number, which contains the highmomentum muon objects. To determine the bunch crossing identification number correctly, the signal delay parameters of all the 320k channels are aligned and the timing for opening the gate is adjusted to the fastest signal according to the result of a clock phase scan with a precision of $1 \mathrm{nsec}$. After the timing calibration and the optimization of trigger conditions, the trigger efficiency is measured as $93.6 \%$ at the plateau region using the muon tracks originating from beam collisions.

In this paper, we report on detailed studies about trigger efficiencies, optimization of coincidence windows and adjustment of gate timing based on the experience of the data acquisition of several months.

KEYWORDS: Trigger concepts and systems (hardware and software); Front-end electronics for detector readout; Wire chambers(MWPC, Thin-gap chambers, drift chambers, drift tubes, proportional chambers etc); Trigger detectors

\footnotetext{
${ }^{1}$ On behalf of ATLAS collaboration
} 


\section{Contents}

1 Level-1 endcap muon trigger 1

2 TGC Clock Phase Scan 3

3 Level-1 Endcap Muon Trigger Efficiency 4

4 Summary 5

\section{Level-1 endcap muon trigger}

The main purpose of the ATLAS level-1 endcap muon trigger system is to select potentially interesting events containing muons with transverse momentum $\left(\mathrm{p}_{T}\right)$ greater than $6 \mathrm{GeV} / \mathrm{c}$ coming from the proton-proton interaction point (IP). If the system finds muon candidates, it sends their coordinates, $\mathrm{p}_{T}$ (classified in six bins) and Bunch Crossing identification number (BCID) to the central trigger system.

The level-1 endcap muon trigger system consists of 3,700 Thin Gap Chambers (TGCs). A chamber has narrow gas gaps of $1.4 \mathrm{~mm}$ between anode wires and cathode strips with $1.8 \mathrm{~mm}$ wire spacing. This structure gives sufficient timing resolution to identify the LHC beam bunch crossing $(25 \mathrm{nsec})$. The readout signals from wires and strips are used to measure the muon hit position in pseudo rapidity $(\eta)$ and the azimuthal angle ( $\phi$ respectively.

The TGC chambers are assembled in three disk structures (stations) for each side and these disks cover $1.05<|\eta|<2.4$. The stations are called TGC1, TGC2, and TGC3 as shown in figure 1. The ATLAS muon system has three large superconducting air-core toroid magnets arranged with an eight-fold azimuthal symmetry and they provide the bending power to determine the muon transverse momentum.

The muon first level trigger is processed by the fully hardware-based system to achieve the design latency of $2.5 \mu \mathrm{sec}$. There are three stages of coincidence operation using signals from all the three TGC stations [1]. The first stage is the 2-station coincidence with signals of TGC2 and TGC3. The second stage is the coincidence between TGC1 and 2-station coincidence, which is called 3-station coincidence. Signals of $\eta$ and $\phi$ are processed independently up to this point. Information of two coordinates is combined in the third stage which is called the $\eta-\phi$ coincidence to make the final decision to identify a track as the level-1 muon trigger candidate.

This $\eta-\phi$ coincidence is performed by electronics named Sector Logic (SL). An SL selects the two highest $\mathrm{p}_{T}$ tracks in each sector. The $\mathrm{p}_{T}$ of the track candidate is determined using the reference table of $\delta \eta$ and $\delta \phi$ which are deviations of the track from the straight (infinite momentum) line drawn from the hit position on TGC3 to the IP in $\eta$ and $\phi$ direction. This reference table shown in figure 2 is named Coincidence Window (CW) which is optimized according to the result of ATLAS full simulation taking into account the magnetic-field map and material in front of the 


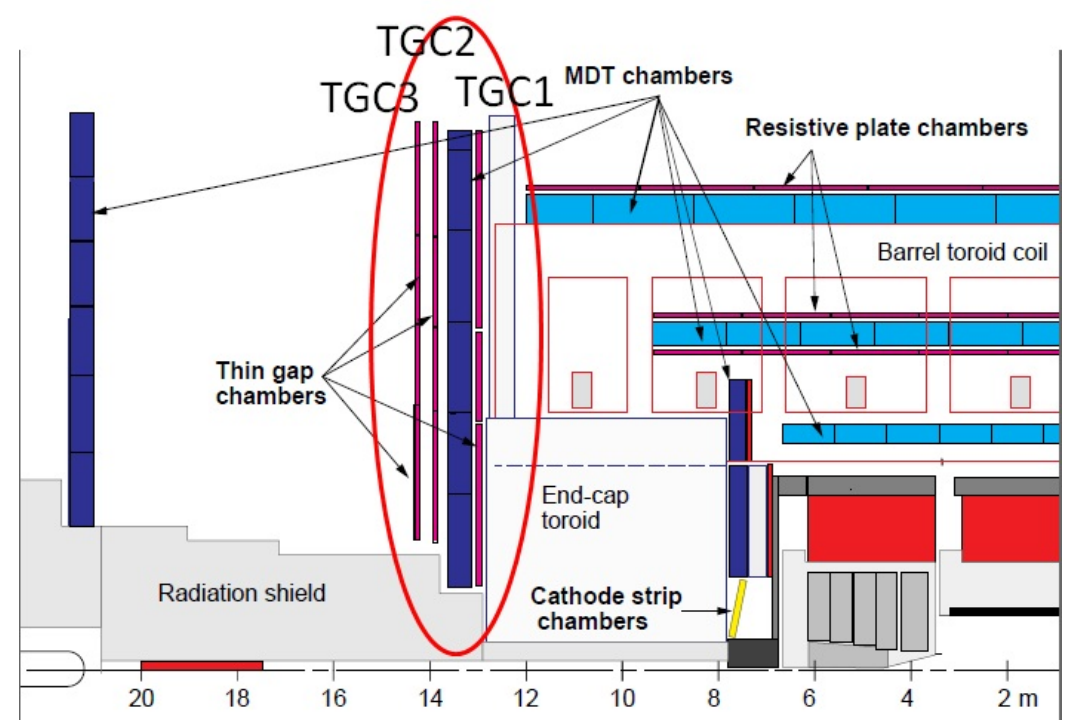

Figure 1. One-quadrant of cross-section in the r-z projection.

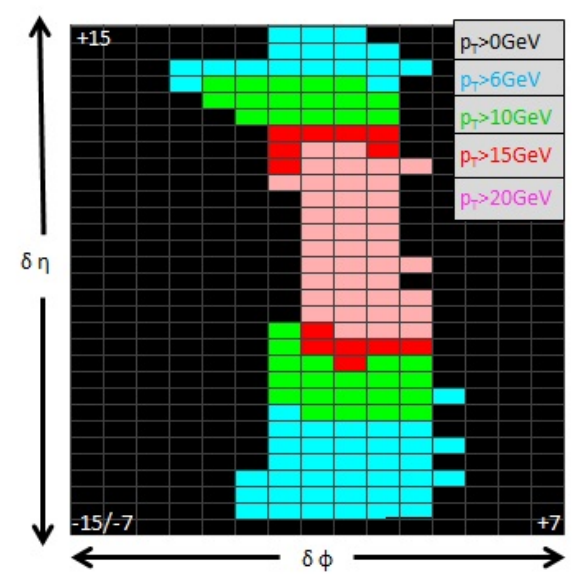

Figure 2. An example of Coincidence-Window (CW). The deviation from the straight track in $v$ is displayed in the horizontal direction and in $\phi$ is displayed in the vertical direction. The different color corresponds to the different trigger-threshold from higher-PT (PT6: 20GeV) to lower-PT (PT1: 6GeV) from center to outwards.

detector. Since the ATLAS magnetic field is not uniform especially in the endcap region, the CWs need to be optimized for each trigger-sector. Introducing 8-fold symmetry of the toroidal field, there are 1,080 types of CWs. The muons having higher transverse-momentum come to the central region of the map, while as the momentum gets smaller the muons move away from the central region.

The $\eta-\phi$ coincidence is processed by referring Look-Up tables (LUTs) stored in the memories of rewritable FPGA (Xilinx Vertex II). The CWs can be updated in order to fit the run condition. Two FPGAs are implemented in a VME 9U board. A FPGA covers a TGC trigger sector and the number of SL boards is 72 for the whole system. 


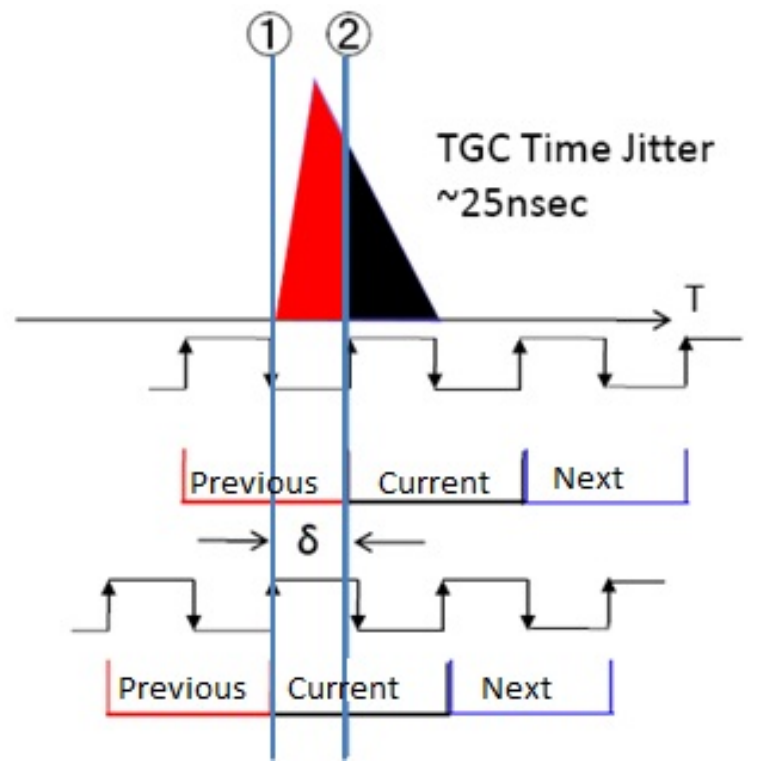

Figure 3. Schematic illustration of the Clock Phase Adjustment.

\section{TGC Clock Phase Scan}

The level-1 endcap muon trigger system has to determine the BCID correctly. In order to trigger properly, all hit signals of a muon in an event need to be caught within the gate with a given BCID. However, TGC signals are intrinsically distributed over the $25 \mathrm{nsec}$ interval. In addition, as cable delays and time-of-flight from the IP varies channel by channel, fine-tuning of gate timing is indispensable. In this section, procedures for adjustment of gate timing are discussed.

The front-end electronics have a delay circuit with a step of $0.8 \mathrm{nsec}$. Delays have been adjusted channel by channel to align all the signals in order to compensate for different cable lengths and time-of-flights. Delay values have been determined using test pulses, which are generated by an embedded test pulse generator. After several iterations with this test pulse adjustment, we could align signals for all the 320k channels within $1.3 \mathrm{nsec}$.

After alignment of the signal timing, we have adjusted gate timing to the collision timing by changing the clock phase. The clock phase has been determined by analyzing collision data containing a good track with $\mathrm{p}_{T}$ greater than $5 \mathrm{GeV} / \mathrm{c}$. At every trigger, the TGC system records hit signals over consecutive three bunches around the bunch to which the trigger signal belongs. The "Current" is the beam collision bunch. The "Previous" and "Next" are earlier and later bunches, respectively. In this condition, current hits should be dominant and no previous hits should be appearing if the gate timing is adjusted correctly. In figure 3 we depicted the adjustment of the gate timing illustratively. A triangle with two colors symbolically expresses several TGC signals recorded (considering time jitter) for a track. If gate timing is not well adjusted, TGC signals can be divided into two bunches. In this example of figure 3, the pulses in red are recorded in the previous bunch while the signals in black are recorded in the current bunch. By changing the clock phase by $\delta$ as shown in figure 3, we could adjust the timing of delay so that all the signals are generated uniquely in the current bunch and no signals in other bunches. The fraction of hits in the previous 


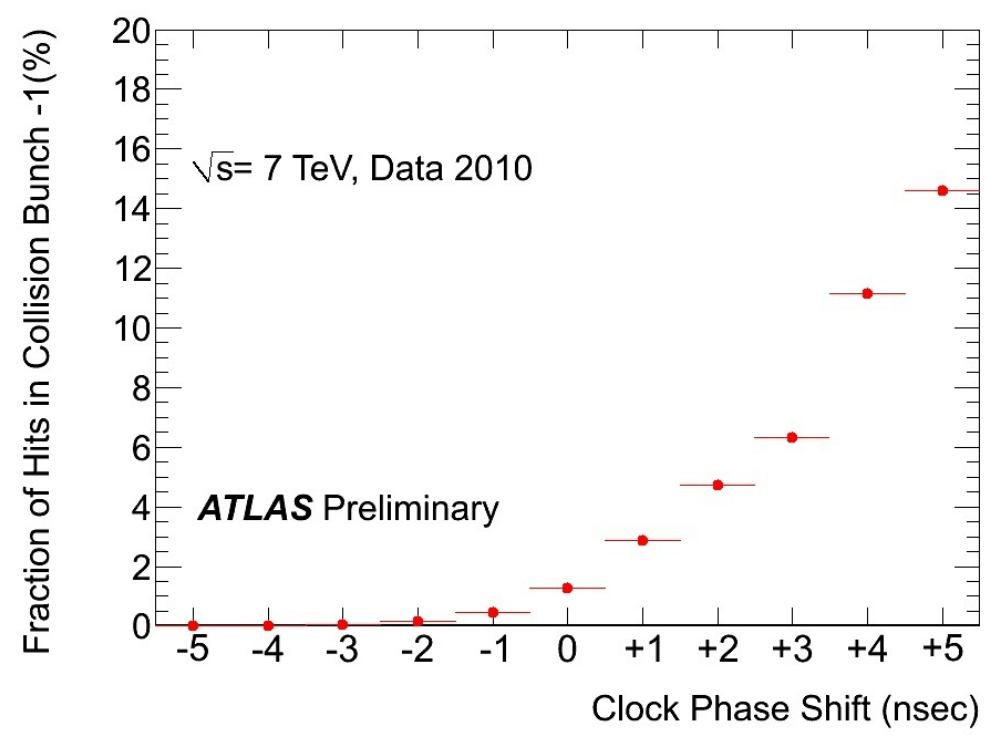

Figure 4. Number of hits observed in the "Previous" bunch over total number of hits observed for a track versus phase shift (delay) of the gate timing.

bunch for all the recorded signals is shown in figure 4 as a function of clock phase timing. We find almost no hit is recorded in the previous bunch if the clock phase is less than -2nsec. The ATLAS clock system has fluctuation of $2 \mathrm{nsec}$ due to the length variations of optical fiber with temperature. ${ }^{1}$ We set clock phase of $-4 n s e c$ to take this effect into account.

\section{Level-1 Endcap Muon Trigger Efficiency}

A trigger item of L1_MU6 is implemented to trigger events which have a muon with $\mathrm{p}_{T}$ greater than $6 \mathrm{GeV} / \mathrm{c}$. The efficiency of L1_MU6 is expected to be $95 \%$ where the $\mathrm{p}_{T}$ is greater than about $15 \mathrm{GeV} / \mathrm{c}$ (plateau region) from the Monte Carlo simulation (The inefficiency is due to large scattering and energy losses in the calorimeter). In reality, the efficiency was measured to be $86 \%$ from data accumulated in the early days of the LHC run due to the difference of cross-talk probability between data and Monte Carlo.

We found that hits in neighboring strip channels can be seen for a muon track. If this cross talk happens on TGC1, the $\delta \phi$ value in the 3 -station coincidence is calculated using the edge channel position of the hit cluster caused by cross-talk, and the result of $\delta \phi$ is different from the original value. This required extending the acceptance region with a wider $\mathrm{CW}$ in the $\delta \phi$ direction than in the case without cross talks. This cross talk effect was estimated to be too small in the MC simulation to optimize the CWs. We have adopted modified CWs with wider acceptance in $\delta \phi$. An example of this modification is shown in figure 5. The original CW in figure 5 (a) is modified as figure 5 (b) taking into account the effect of cross-talk. After this modification, we could observe

\footnotetext{
${ }^{1}$ A clock fiber length between LHC RF and ATLAS is $13.3 \mathrm{~km}$. The time fluctuation of $2 \mathrm{nsec}$ is based on measurements between the LHC bunch arrival time and ATLAS clock during spring and summer 2010.
} 

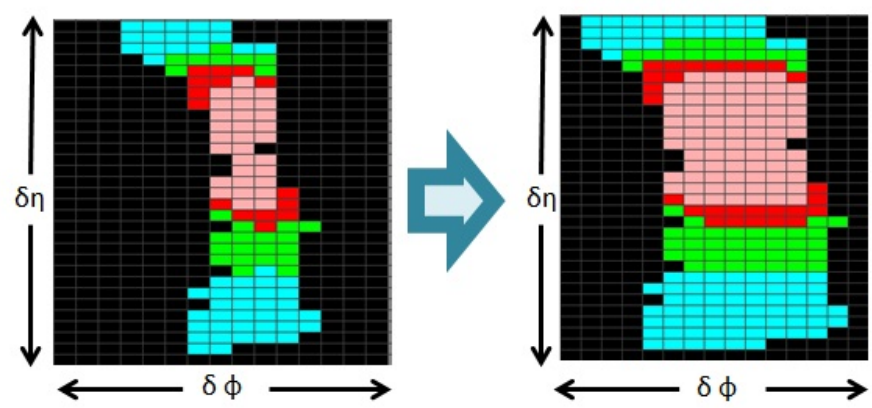

Figure 5. Example of the modification of CW (a): An example of the original CW (b): An optimized CW with the realistic cross-talk probability in the strip channels $(\delta \phi)$.

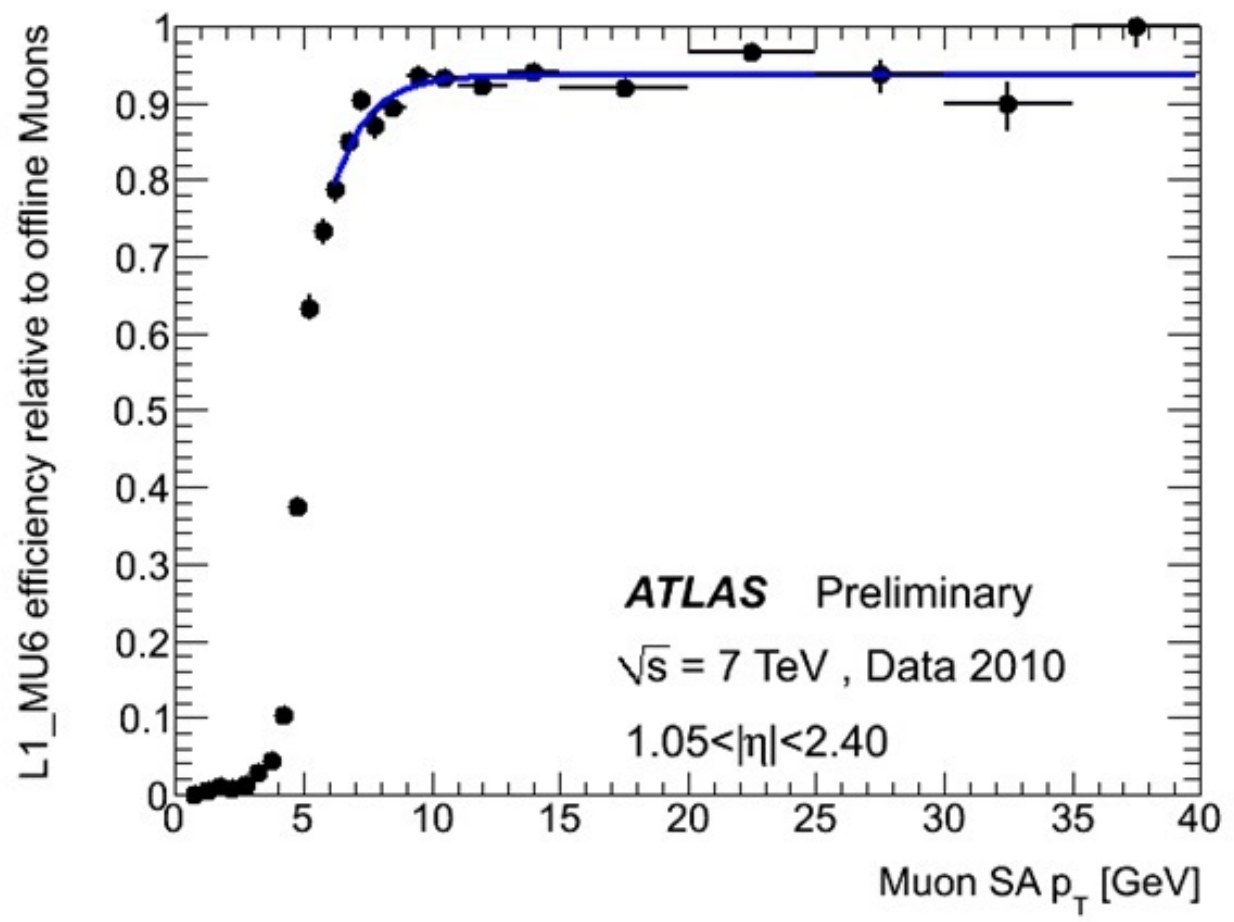

Figure 6. L1_MU6 Efficiency for endcap region.

the improvement of the L1_MU6 efficiency which becomes $93.6 \pm 0.6 \%$ in the plateau region as shown in figure 6.

\section{Summary}

The level-1 endcap muon trigger system is successfully providing trigger signals to the ATLAS central trigger system. We determined the delay parameters using test-pulses. We could align signals for all the channels within $1.3 \mathrm{nsec}$. And the gate timing was adjusted using muons with $\mathrm{p}_{T}$ greater than $5 \mathrm{GeV} / \mathrm{c}$. The timing for opening the gate is adjusted to the fastest signal according to the result of a clock phase scan with a precision of 1nsec. The trigger efficiency of L1_MU6 
is improved from $85 \%$ to $93.6 \%$ by re-estimating the cross-talk probability of strip hits according to the data taken in early LHC runs. Owing to the flexibility of a rewritable FPGA mounted on Sector-Logic, the change can be applied rather smoothly.

\section{References}

[1] ATLAS collaboration, ATLAS Muon Spectrometer Technical Design Report, CERN-LHCC-97-022 (1997). 